\section{Adaptación de los cuestionarios MOS y ComRols a lengua de señas colombiana}

\author{
Adaptation of the questionnaires MOS and \\ ComRols in Colombian sign language
}

\begin{abstract}
Resumen
Objetivo: validar el constructo del apoyo social y adaptar dos cuestionarios en versión de videos a la lengua de señas colombiana. Metodología: estudio psicométrico, $\mathrm{n}=27$ participantes de la comunidad sorda (33,3\% hombres y $66,6 \%$ mujeres), edad media 35,15 (DE 13.467). Instrumentos: cuestionario de apoyo social Medical Outcomes Study (MOS; Sherbourne y Stewart, 1991) y Apoyo a la Comunicación y a la ejecución del Rol Social (ComRols, elaborado por Londoño y Suarez e incluido en el anexo 2 en este artículo) adaptados en versión de videos a lengua de señas colombiana. Procedimiento: el estudio del constructo teórico se realizó con grupos focales y la validez por jurados expertos intérpretes y usuarios de lengua de señas colombiana. Se realizaron análisis psicométricos. Resultados: la evaluación experta reportó puntajes bajos en la estructura gramatical del MOS y puntajes favorables en la prueba ComRols. Análisis psicométrico: Alfa de Cronbah: MOS = .939; Apoyo en comunicación $=.849$ y Apoyo en la ejecución del rol social $=.803$. Validez de criterio de ComRols tomando como referencia el cuestionario MOS = .689 ( $p=.013)$. Conclusiones: los cuestionarios en versión de videos en lengua de señas colombiana son confiables para la comunidad sorda colombiana.
\end{abstract}

\section{Palabras clave}

MOS, apoyo social, apoyo a la comunicación, rol social, lengua de señas, comunidad sorda.

\begin{abstract}
Objective: to validate the construct of social support and adapt two questionnaires in video version to the Colombian sign language. Methodology: psychometric study, $\mathrm{n}=27$ participants from the deaf community (33.3\% men and $66.6 \%$ women), mean age 35.15 (SD 13,467). Instruments: social support questionnaire Medical Outcomes Study (MOS; Sherbourne y Stewart, 1991) and Support for Communication and the Execution of the Social Role (ComRols: authors) adapted in video version to LSC. Procedure: the study of the theoretical construct was carried out with focus groups and the validity by expert juries, interpreters and users of Colombian sign language. Psychometric analyzes were carried out. Results: the expert evaluation reported low scores in the grammatical structure of the MOS and favorable scores in the ComRols test. Psychometric analysis: Cronbah's Alpha: MOS = .939; Support in communication $=.849$ and Support in the execution of the social role $=.803$. ComRols criterion validity taking as reference the questionnaire MOS $=.689(p=$ .013). Conclusions: the questionnaires of the video version of Colombian sign language are reliable for the Colombian deaf community.
\end{abstract}

\section{Keywords}

MOS, social support, communication support, social role, sign language, deaf community.

\author{
Nora Helena Londoño \\ Arredondo \\ <nora.londonno@gmail.com> \\ Universidad San Buenaventura. \\ Colombia
}

\section{Luisa Fernanda Suarez \\ Monsalve \\ <psicologacognitivaluisa@gmail.com>}

Corporación Universitaria Minuto de Dios. Colombia

\section{Yamile Restrepo Restrepo}

<yamilerestrepo1985@gmail.com>

Universidad San Buenaventura.

Colombia

\section{Yeisiri Amaya Montoya \\ <yeisiriamaya44@gmail.com>}

Corporación Universitaria Minuto de Dios. Colombia

\section{Yonathan Alexander \\ Martínez \\ <jonatanm1023@gmail.com>}

Corporación Universitaria Minuto de Dios. Colombia

\section{Damaris Torres Castro}

<dtorrescas5@uniminuto.edu.co>

Corporación Universitaria Minuto de Dios. Colombia

Yolmer Daniel Peña Cordero <ypenacorder@uniminuto.edu.co>

Corporación Universitaria Minuto de Dios. Colombia

\section{Maite Catalina Agudelo Cifuentes \\ <maitecac@gmail.com>}

Universidad CES. Colombia

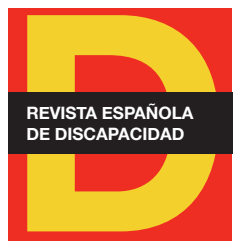

Para citar:

Londoño, N. H. et al. (2021).

Adaptación de los cuestionarios MOS y ComRols a lengua de señas colombiana. Revista Española de Discapacidad, 9(1), pp. 217-237.

Doi: <https://doi.org/10.5569/23405104.09.01.11>

Fecha de recepción: 26-06-2020 Fecha de aceptación: 14-05-2021 


\section{Introducción}

El apoyo social se refiere a la percepción de la disponibilidad de los lazos sociales en caso de ser requerido. Juega un papel significativo en la salud y el bienestar psicológico, y sirve como soporte en el momento de experimentar eventos estresantes relacionados con enfermedad física, problemas emocionales y trastornos mentales (Barrón, 1996). El apoyo social promueve la adaptación positiva a condiciones normales, y contribuye a reducir el efecto negativo del estrés y de las situaciones de riesgo (Barcelata, 2018). Por el contrario, la falta de redes sociales está identificada como uno de los factores que influye en el desencadenamiento de trastornos por estrés postraumático o estrés agudo en personas que han sufrido un evento traumático (Vargas y Coria, 2017) o afectación mayor en las personas que enfrentan enfermedades crónicas (Evon et al., 2011). El apoyo social deficiente es causa de sobrecarga en cuidadores de adultos mayores, lo que genera frecuentemente conflictos familiares y sociales (Da Silva, 2019).

El apoyo social ha sido estudiado ampliamente en el ámbito hospitalario por Sherbourne y Stewart (1991). Estos autores construyeron el cuestionario Medical Outcomes Study Social Support Scale MOS-SSS, en el que se evalúa tanto el apoyo social estructural (cantidad de personas que conforman la red de apoyo social) como el funcional (utilidad del apoyo social). El cuestionario fue validado en una muestra de 2987 participantes con 18 años o más, con condiciones médicas crónicas y observados durante dos años. El análisis factorial exploratorio para los 19 ítems validó cuatro dimensiones: apoyo emocional/informacional (retroalimentación, orientación e información), apoyo tangible (material o instrumental), apoyo afectivo (amor y afecto), interacción social positiva (actividades sociales de entretenimiento con otras personas), con un alfa de Cronbach de 0.91 (Sherbourne y Stewart, 1991).

Otros estudios han considerado tres dimensiones del apoyo funcional: a) apoyo emocional (expresado a través de empatía y confianza); b) apoyo instrumental (se aporta a la solución de problemas); y c) apoyo informativo (se percibe cuando se obtiene información requerida para resolver un problema) (Antonucci e Israel, 1986; Cobb, 1976; Gottlieb, 1983; House, 1981). Sin embargo, otras investigaciones han considerado solo dos dimensiones: apoyo emocional/informacional y apoyo tangible o instrumental (Martínez et al., 2014; Moser et al., 2012; Shyu et al. 2006).

La validación del instrumento MOS ha sido amplia en diferentes condiciones del ámbito de la salud, pero también se ha validado en población no clínica. El estudio realizado por Baca (2016) en personas con cáncer mostró una alta confiabilidad con el modelo de tres factores (Alfa de Cronbach $=0.94$ ) y explicando el $54.2 \%$ de la varianza. El modelo tuvo un buen ajuste $\mathrm{KMO}=0.887$, Bartlet $=\mathrm{p}<0.01$.

En Vietnam, Khuong et al. (2018) realizaron un estudio para evaluar las propiedades psicométricas del cuestionario en pacientes que asistían a tratamiento con metadona (MMT). Usaron una muestra de 300 participantes. Dicha versión mostró una buena consistencia interna (alfa de Cronbach 0.97), además de una buena validez concurrente. El modelo de cuatro factores tuvo un buen ajuste a los datos: CFI 0.97, TLI 0.97, SRMSR 0.03, RMSEA 0.068.

La adaptación del cuestionario MOS al portugués realizada por Soares et al. (2012) con 200 personas con cáncer y en tratamientos en centros de salud, mostró una estructura de tres factores: apoyo afectivo, interacción social positiva, y apoyo social emocional e informacional, y una consistencia interna de 0.95, y para los factores con valores entre 0.70 y 0.87 . 
En Estados Unidos el estudio realizado por Moser et al. (2012) buscó validar las propiedades psicométricas de una versión corta de ocho ítems del cuestionario MOS modificada (mMOS-SS) y compararlo con el cuestionario de 19 ítems. Dicho estudio tomó datos del estudio de cáncer de mama en Boston $(n=660)$ y Los Ángeles $(n=864)$, además datos de un estudio de desenlaces médicos $(n=1,717)$. Los resultados mostraron una buena consistencia interna. El análisis confirmatorio de la estructura de dos factores mostró un buen ajuste del modelo RMSEA= 0.054-0.074 y la validez de constructo y discriminante fue similar en el cuestionario de ocho ítems a la de 19 ítems.

En México, Martínez et al. (2014) realizaron la validación de la versión castellana del cuestionario MOS en 313 participantes con $\mathrm{VIH}$. El análisis exploratorio arrojó una solución de dos factores que explicaron el $72 \%$ de la varianza. Un primer factor de Apoyo Emocional/Informativo con un alfa de Cronbach de 0.97, y un segundo factor de Apoyo Tangible con un alfa de Cronbach de 0.89. El análisis confirmatorio reafirmó esta estructura con buenos índices de ajuste: $\mathrm{NFI}=.916, \mathrm{RFI}=.903, \mathrm{IFI}=.936, \mathrm{TLI}=.926, \mathrm{CFI}=.936, \mathrm{RMSEA}=.096$ (.086-.106).

En China, el estudio de Shyu et al. (2006), con una muestra de 265 adultos cuidadores de familiares con cáncer en cuatro hospitales de diferentes regiones, reportó dos factores que explicaron el $68.98 \%$ de la varianza: apoyo emocional y apoyo instrumental (tangible).

Robitaille et al. (2018), al analizar las propiedades psicométricas, la estructura factorial y la invarianza en las versiones en inglés y en francés del MOS, en personas de 55 años o mayores en Canadá, reportaron una adecuada consistencia interna de la prueba con un rango entre los factores de .90 a .97 para la versión en inglés y de .93 a .97 para la versión en francés. El análisis factorial confirmatorio identificó índices adecuados para los cuatro factores similares al cuestionario original.

En Italia, Giangrasso y Casale (2014) validaron el MOS en estudiantes universitarios ( $n=485 ; 73 \%$ mujeres; media de edad 21.81+_ 1.52). El análisis factorial confirmatorio validó la estructura de cuatro factores principales con buena consistencia interna: Apoyo emocional e informacional, apoyo instrumental, interacción social positivo y apoyo afectivo; $\mathrm{X} 2 / \mathrm{df}=4.49 ; \mathrm{CFI}=.92 ; \mathrm{TLI}=.91 ; \mathrm{RMSEA}=.080$; alfa de Crombach entre .848 a .939$)$.

En Colombia, la validación del cuestionario MOS realizada por Londoño et al. (2012) en población general con 179 participantes reportó un alfa de Cronbach bastante favorable entre los componentes (.921 a .736). La estructura factorial de cuatro dimensiones fue validada mediante el AFC, el cual mostró índices de bondad de ajuste: $\mathrm{GFI}=.780, \mathrm{AGFI}=.713, \mathrm{RMSEA}=.113, \mathrm{AIC}=566.98, \mathrm{BIC}=707.22$.

Con relación a los grupos de estudio y variables asociadas, un grupo de estudios analizó la relevancia del apoyo social en la disminución del estrés y en la solución de problemas en padres con niños con autismo, síndrome de Down y parálisis cerebral (Mahnaz et al., 2015; Sindeaux et al., 2016).

En el estudio realizado por Arfaee y Hesampour (2016) se identificó la relación significativa entre mindfulness, el apoyo social percibido y la salud mental en madres de niños con dificultades intelectuales. En otro estudio con madres que han tenido experiencias adversas (abuso físico, emocional o sexual en la infancia, violencia doméstica, familias disfuncionales con consumo de sustancias, problemas mentales o con problemas carcelarios), el apoyo social mostró ser un moderador en embarazos de riesgo (Recine et al., 2018).

Thompson et al. (2013) estudiaron el impacto del apoyo social en personas con cáncer de mama en estadio temprano, en 541 pacientes y 542 controles, con edad media de 57.7 años (DT = 10.6). La mayoría de los 
participantes reportaron un alto grado de apoyo social. El grupo control mantuvo estabilidad en las puntuaciones mientras que el grupo caso fue más inestable y con mayor afecto negativo (síntomas de depresión o ansiedad) en personas que no estaban casadas o no tenían pareja, ser blanco. Se concluye la importancia de recomendar intervenciones psicosociales del apoyo social para población clínica.

En China, el instrumento fue adaptado al mandarín y validado en personas con VHI/AIDS en una muestra de 200 participantes, utilizando además el cuestionario de depresión de Beck (BDI-II), el cuestionario de ansiedad Zung (SAS), el cuestionario de estrés social percibido (PSS-10) y el cuestionario de calidad de vida de la Organización Mundial de la Salud (WHOQOL-BREF). La consistencia interna de la prueba fue de alfa de Cronbach de 0.97, para los factores con valores entre 0.82 y 0.91 . Las correlaciones fueron negativas con el BDI-II ( $r=-0.41, p<0.01)$; con el SAS $(r=-0.30, p<0.01)$, y correlación significativa y positiva con el WHOQOL ( $r=0.61, p<0.01)$ (Yu et al., 2015).

También en China, en una muestra clínica con 110 participantes, se identificó una correlación significativa entre el cuestionario de calidad de vida con el apoyo social percibido $(r=.82)$, y una correlación negativa con los cuestionarios de ansiedad y depresión $(r=-.58)$. El análisis factorial confirmatorio reportó un alfa de Cronbach de .98, y el rango entre los factores entre .93 - .96 (Yu et al., 2004).

De manera similar, el apoyo social se evaluó en el estado de salud relacionado con la calidad de vida en mujeres con cáncer de seno en un estudio longitudinal con 412 mujeres, utilizando la prueba MOS-SSS y HRQOL para valorar el grado de apoyo social y el estado de salud tres años antes y tres años después de haber recibido el diagnóstico. Las puntuaciones en apoyo social se mantuvieron estables con relación al tiempo, alcanzando a ser una variable que predijo mejorías del estado físico y mental relacionado con la calidad de vida tres años después (Leung et al., 2014).

En personas con problemas cardiovasculares $(n=280)$ se analizó el papel de tres tipos de apoyo: el instrumental, el emocional y asistencial con el autocuidado, en un estudio longitudinal con evaluaciones línea base, a los tres y a los seis meses. Se encontró alta asociación entre el apoyo social y el autocuidado (Fivecoat et al., 2018). De la misma manera, se encontró la asociación entre el apoyo social y conductas de autocuidado $(p=.001 ; r=0.481)$ en la población clínica con problemas cardiovasculares en una muestra de 64 participantes. El apoyo de la familia fue el más relevante $(p=0.001 ; r=0.462)$.

Otro estudio concluye también la importancia del apoyo familiar para generar conductas de autocuidado en la población con problemas coronarios (Khaledi et al., 2014). Y en un estudio realizado con paciente rehospitalizados se encontraron niveles bajos de apoyo social $(\mathrm{t}=-4.007, \mathrm{df}=211, \mathrm{p}=<.001)$ y autocuidado $(\mathrm{t}=-3.343$, $d f=220, p=<.002)$ (Chamberlai, 2017).

El apoyo social a través de la facilitación en la comunicación ha sido estudiado en comunidades migrantes que viven lejos de sus familias. La calidad, la cantidad y la disposición de la comunicación ha sido considerada como un factor protector para mantener la red de apoyo (Juárez, 2008). Sin embargo, no ha sido estudiado en la comunidad sorda ni se encontraron cuestionarios para evaluarlo. Tampoco se encontró la adaptación del cuestionario MOS a lengua de señas colombiana (LSC). Si bien el cuestionario inicialmente fue creado en contexto hospitalario para conocer la percepción de apoyo social y su relación con la recuperación, algunos estudios han realizado la validación en población no clínica (Giangrasso y Casale, 2014; Londoño et al., 2012). En la comunidad sorda, población no clínica, se considera de igual manera útil adaptar el cuestionario a lengua de señas, en este caso a lengua de señas colombiana. 
Teniendo en cuenta las razones fundamentales que plantea Chahín-Pinzón (2014) por las cuales se debe llevar a cabo la adaptación de un cuestionario, haciendo referencia a los aspectos prácticos (agilidad y economía) y la necesidad de interpretar y comparar los resultados en diferentes culturas, se consideró que la comunidad sorda podría beneficiarse de la adaptación del cuestionario MOS a lengua de señas.

El proceso de adaptación conlleva un estudio cultural y son clave los comités de expertos para conseguir que el instrumento resultante mantenga la equivalencia semántica, idiomática, conceptual y experiencial con el cuestionario original (Hyrkäs et al., 2003; Mikulic, 2015; Ramada et al., 2013), en especial frente a las dificultades que enfrenta la comunidad sorda al presentar pruebas en español escritas cuando se utilizan metáforas (Naranjo y Guerrero, 2013). El presente estudio tomó en cuenta los comités de expertos en diferentes momentos de la investigación tal como se describe en el procedimiento.

En el caso de la comunidad sorda colombiana la lengua es la lengua de señas colombiana, caracterizado por ser visual, gestual y espacial, con su propio vocabulario, expresiones idiomáticas, gramáticas y sintaxis diferentes del español. Los elementos de la lengua son la configuración, posición y orientación de las manos en relación con el cuerpo y con el individuo, el espacio, dirección y velocidad de movimientos, así como la expresión facial (Congreso de la República, 2005; Ley 982, 2015, Art 1\%). La persona sorda se define como aquella "que no posee la audición suficiente y que en algunos casos no puede sostener una comunicación y socialización natural y fluida en lengua oral alguna, independientemente de cualquier evaluación audiométrica que se le pueda practicar" (Congreso de la República, 2005; Ley 982, 2015, Art. 1\%).

Por otra parte, al considerar otras necesidades específicas con las cuales cuenta la población de estudio, y que no incluye el cuestionario MOS, como la necesidad para comunicarse con la población en general y de ejercer su rol social (académico y/o laboral), se adicionaron estos dos componentes en un nuevo cuestionario.

Teniendo en cuenta lo anterior, la investigación se planteó como objetivo general validar el constructo del apoyo social y adaptar dos cuestionarios en versión de videos a la lengua de señas colombiana. De esta manera, se aporta a la comunidad sorda al disponer de dos cuestionarios para su uso en lengua de señas colombiana.

\section{Metodología}

Estudio psicométrico que buscó adaptar los cuestionarios a lengua de señas colombiana.

\subsection{Participantes}

La muestra estuvo conformada por 27 personas sordas (33.3\% hombres y $66.6 \%$ mujeres), edad media 35.15 (DE 13.47). La selección fue por conveniencia y se acudió a instituciones de educación básica y superior y la Asociación Antioqueña de Personas Sordas (ASANSO), para aplicar el cuestionario de manera individual. 


\subsection{Instrumentos}

Medical Outcomes Study (MOS) (Sherbourne y Stewart, 1991). Evalúa el apoyo social percibido. Cuenta con 20 ítems; el primero hace referencia al tamaño de la red y evalúa el apoyo social estructural, y los 19 ítems restantes evalúan el apoyo social funcional en cuatro dimensiones: apoyo emocional/informacional (ítems $3,4,8,9,13,16,17$, y 19), apoyo instrumental (ítems 2, 5, 12 y 15), interacción social positiva (ítems 7, 11, 14 y 18) y apoyo afectivo (ítems 6, 10 y 20) (anexo 1). Las opciones de respuesta de estos 19 ítems están en escala Likert de 1 (nunca) a 5 (siempre). En el estudio de Londoño et al. (2012) en población general colombiana se encontró un índice de confiabilidad del cuestionario de alfa de Cronbach de .941 y entre los factores de .736 a .921. El análisis factorial confirmatorio mostró índices de bondad de ajuste: GFI = .780, $\mathrm{AGFI}=.713, \mathrm{RMSEA}=.113, \mathrm{AIC}=566.98, \mathrm{BIC}=707.22$.

Cuestionario de apoyo en la comunicación y en la ejecución del rol social (ComRols, incluido en el anexo 2): evalúa el apoyo social en la comunicación a través de 7 ítems y el apoyo social para la ejecución del rol social a través de 5 ítems, opciones de respuesta de 1 (nunca) a 3 (siempre) (anexo 2).

En el anexo 3 se especifican los enlaces de cada ítem en lengua de señas colombiana.

\subsection{Procedimiento}

Se estudió en el contexto de la comunidad sorda colombiana la equivalencia del constructo teórico de apoyo social percibido utilizado por Sherbourne y Stewart (1991). Se acudió a grupos focales de discusión conformados por la misma comunidad sorda, intérpretes y familiares usuarios de lengua de señas colombiana. Una vez comprendida la definición de apoyo social desde el punto de vista de los participantes en los grupos de discusión y analizada la equivalencia y aplicabilidad, se validó el constructo pero con la necesidad de ampliarlo al apoyo en la comunicación y en la ejecución del rol social (académico/laboral).

Estableciendo la equivalencia, uno de los talleres realizados en los grupos focales tuvo como resultado la elaboración de ítems. Dentro de este banco de ítems se identificó un grupo de estos que evaluaban el apoyo a la comunicación y a la ejecución del rol social (académico/laboral) y se consolidó el nuevo cuestionario.

Se inició una segunda fase donde se reestructuró la gramática y se interpretaron los ítems a lengua de señas colombiana en versión de videos. Luego se reinterpretaron a la versión del idioma castellano escrito. Esta actividad se realizó con intérpretes oyentes con dominio teórico tanto en lengua castellana como en teoría gramatical de lengua de señas colombiana.

Los videos finales fueron evaluados por tres jurados expertos externos a la investigación, intérpretes oyentes usuarios de lengua de señas colombiana, buscando comparar la correspondencia teórica entre los ítems planteados para ambos cuestionarios. Se estableció el consenso entre los investigadores y los expertos respecto a la pertinencia de cada ítem en torno al constructo de apoyo social en población sorda. La recopilación de información se realizó mediante una matriz en donde se proponía a los expertos calificar la equivalencia semántica y pertinencia en la traducción/interpretación de los ítems del MOS, y los que conforman el nuevo cuestionario ComRols, entre tres opciones de selección, con la posibilidad de hacer recomendaciones.

Respecto a las dimensiones del nuevo cuestionario ComRols, los jurados debían además calificar cada ítem en una escala de cuatro opciones de selección, la claridad (fácil comprensión del ítem) y relevancia 
(preeminencia o importancia del ítem), la coherencia (con relación a las nuevas categorías del apoyo social) y la suficiencia (los ítems de cada dimensión bastan para su medición), y con una escala de tres opciones la pertinencia de las nuevas escalas ( $1=$ Nunca a $3=$ Siempre).

Con la información sistematizada se procedió a calcular la concordancia entre jurados usando métodos estadísticos de análisis descriptivos (medias, desviaciones y promedios) y correlacionales para identificar el alfa de Cronbach (escala de confiabilidad), y finalmente elaborar las conclusiones del juicio de expertos que serían usadas en la descripción psicométrica de la prueba.

Para el trabajo de campo propiamente dicho, se presentó el consentimiento informado y luego los dos cuestionarios en la versión de video. Los investigadores con dominio en lengua de señas colombiana apoyaron el proceso de evaluación. Con las respuestas reportadas se elaboró la base de datos y se realizaron los análisis psicométricos.

\subsection{Consideraciones éticas}

Se elaboró el consentimiento informado para los participantes sordos en LSC. Desde la Ley Colombiana 1090 de 2006, en el presente estudio se da cumplimiento a los artículos 50 y 55 . En relación con la Resolución 8430 de 1993 del Ministerio de Salud, se presenta un riesgo mínimo, dando cumplimiento a los artículos 4, 5, 6, 8, 9, 10, 12, 14 y 26 . Se ajustó a los principios éticos necesarios para el estudio.

\section{Resultados}

\subsection{Evaluación de expertos}

Para el cuestionario MOS, los jurados evaluaron como insuficiente la pertinencia de la traducción/interpretación de los videos (promedio 1.46, media 29.3, DT 5.8, confiabilidad alfa de Cronbach .85), señalando deficiencias en elementos relacionados con la estructura gramatical en lengua de señas colombiana de los ítems, así como el uso reiterado de la palabra "quien".

Para el cuestionario ComRols, la evaluación de la dimensión de apoyo en la comunicación mostró una tendencia a puntuaciones bajas en claridad (promedio 1.7, media 12, DT 4.3, confiabilidad alfa de Cronbach .982), puntuaciones medias en relevancia (promedio 3.2, media 17.6, DT 4, confiabilidad alfa de Cronbach .47), pertinencia en la escala de respuesta (promedio 2, media 2, DT 0) y en la traducción/interpretación (promedio 2.8, media 20, DT 11.5, confiabilidad alfa de Cronbach -.06), acuñando observaciones de señas que no corresponden al equivalente del concepto en castellano, e impreciso uso de los elementos de la lengua de señas colombiana. Se reportaron puntuaciones altas en coherencia (promedio 3.6, media 3.6, DT .57) y suficiencia (promedio 3.6, media 3.6, DT 2.57).

En el caso de la dimensión de apoyo en la ejecución del rol social, la valoración de jurados arrojó puntuaciones bajas en el componente de claridad (promedio 1.6, media 8.3, DT 2.8, c, que pudo estar mediada por 
la calidad de la traducción/interpretación de acuerdo a lo observado, al igual que en la dimensión anterior) y puntuaciones medias en relevancia (promedio 2.6, media 15, DT 5, confiabilidad alfa de Cronbach .93, con respuestas que indican moderada relevancia), coherencia (promedio 2.6, media 2.6, DT .57), suficiencia (promedio 3.3, media 3.3, DT .57), pertinencia en la escala de respuesta (promedio 2, media 2, DT 0), y pertinencia en la traducción/interpretación (promedio 2, media 10, DT 0) (tabla 1).

\begin{tabular}{|c|c|c|c|c|c|c|}
\hline \multirow{2}{*}{ Variable } & \multirow{2}{*}{ Media } & \multirow{2}{*}{ Desviación } & \multirow{2}{*}{ Promedio } & \multirow{2}{*}{$\begin{array}{l}\text { Alfa de } \\
\text { Cronbach }\end{array}$} & \multicolumn{2}{|c|}{$\begin{array}{c}\text { Intervalo de confianza } \\
95 \% \\
\end{array}$} \\
\hline & & & & & $\begin{array}{l}\text { Límite } \\
\text { inferior }\end{array}$ & $\begin{array}{l}\text { Límite } \\
\text { superior }\end{array}$ \\
\hline \multicolumn{7}{|c|}{ Comunicación } \\
\hline Claridad & 12 & 4.3 & 1.7 & .982 & .59 & .99 \\
\hline Relevancia & 17.6 & 4 & 3.2 & .47 & -.098 & .91 \\
\hline Coherencia & 3.6 & .57 & & & & \\
\hline Suficiencia & 3.6 & .57 & & & & \\
\hline $\begin{array}{l}\text { Pertinencia en escala de } \\
\text { respuesta }\end{array}$ & 2 & 0 & & & & \\
\hline $\begin{array}{l}\text { Pertinencia en la } \\
\text { Traducción/Interpretación }\end{array}$ & 20 & 11.5 & 2.8 & -.06 & .13 & .83 \\
\hline \multicolumn{7}{|c|}{ Rol social } \\
\hline Claridad & 8.3 & 2.8 & 1.6 & 1 & 1 & 1 \\
\hline Relevancia & 15 & 5 & 2.6 & .93 & .22 & .99 \\
\hline Coherencia & 2.6 & .57 & & & & \\
\hline Suficiencia & 3.3 & .57 & & & & \\
\hline $\begin{array}{l}\text { Pertinencia en escala de } \\
\text { respuesta }\end{array}$ & 2 & 0 & & & & \\
\hline $\begin{array}{l}\text { Pertinencia en la } \\
\text { Traducción/Interpretación }\end{array}$ & 10 & 0 & 2 & - & - & - \\
\hline \multicolumn{7}{|c|}{ MOS } \\
\hline $\begin{array}{l}\text { Pertinencia en la } \\
\text { Traducción/Interpretación }\end{array}$ & 29.3 & 5.8 & 1.46 & .85 & .03 & .93 \\
\hline
\end{tabular}

Fuente: elaboración propia.

\section{- Análisis estadístico de ítems MOS y ComRols}

Inicialmente se realizó el análisis de los ítems de los cuestionarios MOS. Se pudo observar que todas las desviaciones estuvieron por encima de 1 , reflejando una buena variabilidad. Los promedios de la mayor parte de los puntajes oscilaron entre 2 y 3 , que era lo esperado considerando que la escala puntúa entre 1 y 5. Además, se hizo el análisis de ítems para el cuestionario ComRols, escala que se puntúa entre 1 y 3 , encontrando poca variabilidad. Las desviaciones de todos los ítems estuvieron por debajo de 1 (tabla 2). 


\begin{tabular}{|c|c|c|c|c|c|c|c|c|}
\hline Variable & $\mathbf{N}$ & Perdidos & Media & Mediana & Desv. típ. & Varianza & Mínimo & Máximo \\
\hline mos2 & 24 & 1 & 3.6 & 4.0 & 1.4 & 1.9 & 1 & 5 \\
\hline mos3 & 25 & 0 & 3.0 & 3.0 & 1.5 & 2.3 & 1 & 5 \\
\hline mos 4 & 23 & 2 & 2.9 & 3.0 & 1.3 & 1.8 & 1 & 5 \\
\hline mos5 & 25 & 0 & 3.4 & 4.0 & 1.7 & 2.8 & 1 & 5 \\
\hline mos6 & 23 & 2 & 3.8 & 4.0 & 1.5 & 2.3 & 1 & 5 \\
\hline mos7 & 25 & 0 & 2.9 & 3.0 & 1.4 & 2.0 & 1 & 5 \\
\hline mos8 & 21 & 4 & 2.9 & 3.0 & 1.5 & 2.4 & 1 & 5 \\
\hline $\operatorname{mos} 9$ & 24 & 1 & 2.5 & 2.0 & 1.3 & 1.7 & 1 & 5 \\
\hline $\operatorname{mos} 10$ & 24 & 1 & 3.2 & 3.0 & 1.6 & 2.4 & 1 & 5 \\
\hline $\operatorname{mos} 11$ & 19 & 6 & 2.6 & 2.0 & 1.4 & 2.0 & 1 & 5 \\
\hline $\operatorname{mos} 12$ & 25 & 0 & 3.2 & 3.0 & 1.5 & 2.1 & 1 & 5 \\
\hline $\operatorname{mos} 13$ & 21 & 4 & 3.0 & 3.0 & 1.3 & 1.7 & 1 & 5 \\
\hline $\operatorname{mos} 14$ & 20 & 5 & 2.6 & 2.0 & 1.2 & 1.5 & 1 & 5 \\
\hline $\operatorname{mos} 15$ & 25 & 0 & 3.2 & 3.0 & 1.5 & 2.3 & 1 & 5 \\
\hline $\operatorname{mos} 16$ & 24 & 1 & 2.9 & 2.5 & 1.4 & 2.0 & 1 & 5 \\
\hline $\operatorname{mos} 17$ & 22 & 3 & 3.1 & 3.0 & 1.3 & 1.6 & 1 & 5 \\
\hline $\operatorname{mos} 18$ & 24 & 1 & 3.2 & 3.0 & 1.4 & 1.9 & 1 & 5 \\
\hline $\operatorname{mos} 19$ & 21 & 4 & 3.0 & 3.0 & 1.4 & 1.8 & 1 & 5 \\
\hline $\operatorname{mos} 20$ & 23 & 2 & 3.6 & 4.0 & 1.6 & 2.5 & 1 & 5 \\
\hline com1 & 24 & 1 & 2.2 & 2.0 & 0.8 & 0.6 & 1 & 3 \\
\hline com2 & 24 & 1 & 2.3 & 2.0 & 0.6 & 0.4 & 1 & 3 \\
\hline com3 & 22 & 3 & 1.9 & 2.0 & 0.8 & 0.6 & 1 & 3 \\
\hline com4 & 24 & 1 & 1.8 & 2.0 & 0.8 & 0.6 & 1 & 3 \\
\hline com5 & 24 & 1 & 2.0 & 2.0 & 0.6 & 0.4 & 1 & 3 \\
\hline com6 & 22 & 3 & 2.2 & 2.0 & 0.5 & 0.3 & 1 & 3 \\
\hline com7 & 23 & 2 & 1.8 & 2.0 & 0.7 & 0.4 & 1 & 3 \\
\hline rols1 & 20 & 5 & 1.7 & 2.0 & 0.7 & 0.5 & 1 & 3 \\
\hline rols2 & 24 & 1 & 1.8 & 2.0 & 0.7 & 0.4 & 1 & 3 \\
\hline rols3 & 25 & 0 & 1.6 & 2.0 & 0.7 & 0.5 & 1 & 3 \\
\hline rols4 & 24 & 1 & 1.6 & 1.5 & 0.7 & 0.5 & 1 & 3 \\
\hline rols5 & 22 & 3 & 1.9 & 2.0 & 0.8 & 0.6 & 1 & 3 \\
\hline
\end{tabular}

Fuente: elaboración propia.

El puntaje promedio del cuestionario MOS fue de 60.5 (DE 19.1). El valor mínimo fue de 19 y el máximo de 95. En la dimensión de apoyo emocional/informacional el valor promedio fue de 23.5 (DE 8.9), en la dimensión de apoyo instrumental el valor promedio fue de 13.5 (DE 5.1), la interacción social positiva tuvo un 
promedio de 11.9 (DE 4.7), y el valor promedio de apoyo afectivo fue de 11 (DE 3.7). Por otro lado, el puntaje promedio obtenido por los participantes para el cuestionario ComRols fue de 22.8 (DE 5.5). La dimensión de apoyo en la comunicación fue de 14.4 (DE 3.5) y la dimensión de apoyo en la ejecución del rol social tuvo un puntaje promedio de 8.6 (DE 2.6) (tabla 3).

Tabla 3. Estadísticos descriptivos para los puntajes de las dimensiones de los cuestionarios MOS y ComRols

\begin{tabular}{|l|c|c|c|c|c|c|}
\hline Items & Media & Mediana & Desv. típ. & Varianza & Mínimo & Máximo \\
\hline Apoyo emocional informacional & 23.5 & 21.5 & 8.9 & 78.9 & 8 & 40 \\
\hline Apoyo Instrumental & 13.5 & 14.5 & 5.1 & 26.3 & 4 & 20 \\
\hline Interacción social positiva & 11.9 & 11.5 & 4.7 & 21.8 & 4 & 20 \\
\hline Apoyo afectivo & 11.0 & 12.0 & 3.7 & 14.0 & 3 & 15 \\
\hline Puntaje total MOS & 60.5 & 58.0 & 19.1 & 364.8 & 19 & 95 \\
\hline Apoyo en la comunicación & 14.4 & 14.0 & 3.5 & 12.4 & 7 & 21 \\
\hline Apoyo en la ejecución del rol social & 8.6 & 8.0 & 2.6 & 6.6 & 5 & 15 \\
\hline Puntaje total ComRols & 22.8 & 23.0 & 5.5 & 30.8 & 12 & 36 \\
\hline
\end{tabular}

Fuente: elaboración propia.

Se evaluó la consistencia interna del cuestionario MOS de acuerdo con las dimensiones propuestas por los autores del cuestionario original, encontrando que el cuestionario tuvo una buena consistencia interna (alfa de Crombach 0.94). Por su parte, las dimensiones mostraron valores satisfactorios, la dimensión con mayor consistencia fue emocional informacional (tabla 4).

\begin{tabular}{|c|c|c|}
\hline Cuestionario & Dimensión & $\begin{array}{c}\text { Alfa de } \\
\text { Crombach }\end{array}$ \\
\hline \multirow{5}{*}{ MOS } & MOS total & 0.939 \\
\hline & Apoyo emocional/informacional & 0.927 \\
\hline & Apoyo instrumental & 0.861 \\
\hline & Interacción social positiva & 0.831 \\
\hline & Apoyo afectivo & 0.795 \\
\hline \multirow{3}{*}{ ComRols } & ComRol Total & 0.891 \\
\hline & Apoyo en la comunicación & 0.849 \\
\hline & Apoyo en la ejecución del rol social & 0.803 \\
\hline
\end{tabular}

Fuente: elaboración propia.

Al realizar los análisis comparativos del cuestionario MOS con esta muestra en relación con la población normal, se encontraron puntuaciones más bajas en la muestra estudiada (tabla 5). 


Tabla 5. Análisis descriptivos y comparativos de los cuestionarios MOS
\begin{tabular}{|l|c|c|c|c|c|c|}
\hline & $\begin{array}{c}\text { Comunidad } \\
\text { sorda } \\
\mathbf{n}=\mathbf{2 7} \\
\text { Media (DT) }\end{array}$ & $\begin{array}{c}\text { Población } \\
\text { general } \mathbf{n = 1 7 9} \\
\text { Media (DT) }\end{array}$ & $\mathbf{t}$ & $\boldsymbol{p}$ & $\mathbf{L i}$ & Is \\
\hline Apoyo emocional/informacional & $23.5(8.9)$ & $32.8(6.6)$ & -4.62 & .000 & -13.33 & -4.99 \\
\hline Apoyo instrumental & $13.5(5.1)$ & $16.6(3.4)$ & -3.20 & .004 & -5.09 & -1.10 \\
\hline Interacción social positiva & $11.9(4.7)$ & $16.5(3.3)$ & -3.96 & .001 & -6.64 & -2.02 \\
\hline Apoyo afectivo & $11(3.7)$ & $12.7(2.5)$ & -2.94 & .033 & -3.42 & -.16 \\
\hline
\end{tabular}

Fuente: elaboración propia.

Se pudo identificar validez de criterio para el cuestionario ComRols, tomando como referente el cuestionario MOS. Esto se determinó a partir de las correlaciones entre los puntajes de los cuestionarios. El coeficiente de correlación entre el puntaje total de MOS-SSS y el puntaje total de ComRol fue de 0.689 ( $p=0.013$ ) (tabla 6).

\begin{tabular}{l}
\hline Tabla 6. Correlaciones entre los puntajes de los cuestionarios MOS y ComRols \\
\begin{tabular}{|l|c|c|}
\hline Cuestionarios & $\begin{array}{c}\text { Coeficiente de } \\
\text { correlación }\end{array}$ & P \\
\hline MOS total - Apoyo social ComRols Total & 0.689 & 0.013 \\
\hline MOS total - Com & 0.659 & 0.02 \\
\hline MOS total - Rols & 0.657 & 0.008 \\
\hline
\end{tabular}
\end{tabular}

Fuente: elaboración propia.

El análisis factorial exploratorio no pudo ser realizado dado que el tamaño de la muestra estuvo por debajo de 30 participantes.

\section{Discusión}

El aporte del estudio giró en torno a tres aspectos fundamentales: primero, la necesidad de ampliar la conceptualización del constructo de apoyo social a la comunidad sorda colombiana; segundo, al proceso de adaptación y validación de los cuestionarios a lengua de señas colombiana; y tercero, a la evaluación de la percepción del apoyo social de la comunidad sorda evaluada. 
En relación con la necesidad de ampliar el constructo del apoyo social en la comunidad sorda, las dimensiones en general se han centrado en el componente funcional del apoyo social, identificando cuatro dimensiones básicas: apoyo afectivo (amor y afecto), emocional/informacional (retroalimentación, orientación), interacción social positiva (actividades sociales de entretenimiento con otras personas) e instrumental (tangible, material, económico) (Sherbourne y Stewart, 1991). Para la comunidad sorda se validaron dichas dimensiones y se adicionaron dos respecto al apoyo en la comunicación y en la ejecución del rol social (académico/ laboral). El apoyo en la comunicación se expresa en la necesidad de que otras personas se comuniquen con ellos en lengua de señas colombiana. Personas que les expliquen cuando no entienden lo que ocurre, alerten del peligro, faciliten la comunicación con otros, interpreten lo que otras personas dicen y les pregunten qué quieren o necesitan hacer en el día. El apoyo en la ejecución del rol social está representado en el apoyo académico o laboral y el aprovechamiento de las oportunidades que benefician la calidad de vida.

En segundo lugar, respecto al proceso de adaptación y validación de los cuestionarios a lengua de señas colombiana, estos reportaron una alta confiabilidad en general. Si bien los jurados expertos evaluaron de manera deficiente la pertinencia en la traducción/interpretación del cuestionario MOS, críticas que también se han realizado en otros intentos de traducir pruebas del español a lengua de señas por hacer parte de otra cultura y otra tradición ajena a la cultura sorda y a la lengua de señas colombiana (Naranjo y Guerrero, 2013), los análisis estadísticos para las cuatro dimensiones reportaron adecuada confiabilidad, consistencia y validez concurrente entre los cuestionarios. Esto hace suponer que, si bien se puede mejorar la estructura de los enunciados en lengua de señas colombiana, la estructura psicométrica es adecuada, tal como en general lo reportan otros estudios para cuatro dimensiones (Giangrasso y Casale, 2014; Khuong et al., 2018; Londoño et al., 2012; Robitaille et al., 2018; Sherbourne y Steward, 1991).

Por último, la evaluación del nivel de apoyo social con el que cuenta la comunidad sorda (MOS; Sherbourne y Stewart, 1991, adaptado a lengua de señas colombiana por Londoño et al., 2012) comparada con población normal colombiana (Londoño et al., 2012), identificó niveles significativamente inferiores en la comunidad sorda. Esto puede interpretarse por la limitación del apoyo social y la mediación necesaria del intérprete en la comunicación, quien no siempre hace parte de su grupo familiar o amigos. Por otro lado, se identifica la falta de apoyo en el contexto educativo, laboral, familiar y gubernamental, tal como lo expresaron los participantes en los grupos focales del estudio. El ámbito hospitalario es valorado por la comunidad sorda como uno de los sectores interesados en comunicarse pese al poco dominio de la lengua de señas colombiana. Consideran algunos que sus familiares no siempre se muestran interesados en aprender lengua de señas colombiana y limitan la socialización con la comunidad sorda. Lo anterior confirma la necesidad de atender las necesidades de apoyo social en esta comunidad (Quiceno y Arbeláez, 2016), y así favorecer procesos de adaptación y resiliencia (Barcelata, 2018), mejorando las estrategias de afrontamiento (Rutter, 2007), autoeficacia (Bandura, 2009) y regulación emocional (Grass, 2013) de la comunidad sorda.

\section{Conclusiones}

El procedimiento permitió dar cumplimiento al objetivo general, establecer la equivalencia del constructo teórico del apoyo social en la cultura sorda que permitiera adaptar el cuestionario de apoyo social percibido MOS a lengua de señas colombiana. 
Se considera pertinente hacer la modificación del cuestionario donde se identifique la percepción del apoyo social no solo en el ámbito hospitalario sino académico, familiar, educativo, laboral y gubernamental, que puede llegar a ser validado para la comunidad a partir de la reflexión en relación con el desarrollo de la lengua de señas colombiana en nuestro país. Es ideal que las herramientas psicométricas sean aplicadas a la comunidad sorda respetando su autonomía lingüística, dado que la lengua de señas colombiana no es igual en gramática y variedad de vocabulario que el español. Tener la herramienta de video adaptada no es suficiente; se requiere de un intérprete capacitado en el cuestionario o en el mejor de los casos, un psicólogo usuario de lengua de señas colombiana conocedor del cuestionario, para atender a que se mantenga el sentido y el significado de la pregunta. Por último, teniendo en cuenta los resultados de los análisis comparativos, se recomienda reforzar el apoyo familiar, social y de la comunidad en general para la población sorda.

\section{- Limitaciones}

La adaptación lograda no constituye un ajuste razonable para toda la población sorda y la adaptación fue realizada específicamente para Colombia.

La adaptación del cuestionario podría ser un paso para la validación, sin desconocer las barreras de la interpretación dadas las diferencias culturales y la tradición oral en la que fueron construidas las preguntas de los cuestionarios. 


\section{Referencias bibliográficas}

Antonucci, T. C. e Israel, B. A. (1986). Veridicality of social support: a comparison of principal and network members' responses. Journal of Consulting and Clinical Psychology, 54, pp. 432-437.

Arfaee, F. S. y Hesampour, F. (2016). The relationship between mindfulness and perceived social support and mental health in mothers of children with intellectual disability. Socialworkmag, 4(4), pp. 42-52.

Baca, D. (2016). Confiabilidad y validez del cuestionario de apoyo social en pacientes con cáncer de Trujillo. Revista de Investigación en Psicología, 19(1), pp. 177-190.

Bandura, A. (2009). Social cognitive theory goes global. The Psychologist, 22(6), pp. 504-506.

Barcelata, B. E. (2018). Adaptación y resiliencia adolescente en contextos múltiples. Manual Moderno.

Barrón, A. (1996). Apoyo social. Aspectos teóricos y aplicaciones. Siglo Veintiuno Editores, S.A.

Chahín-Pinzón, N. (2014). Aspectos a tener en cuenta cuando se realiza una adaptación de test entre diferentes culturas. Psychologia: avances de la disciplina, 8(2), pp. 109-112. http://www.scielo.org.co/pdf/psych/v8n2/ v8n2a09.pdf.

Chamberlai, L. (2017). Perceived social support and self-care in patients hospitalized with heart failure. European Journal of Cardiovascular Nursing, 16(8), pp. 753-761. https://doi.org/10.1177/1474515117715842.

Cobb, S. (1976). Social support as a moderator of life stress. Psychosomatic Medicine, 38, pp. 300-314.

Da Silva, C. Y. (2019). Ser cuidador. Estrategias para el cuidador del adulto mayor. Manual Moderno.

Evon, D. M. et al. (2011). Social support and clinical outcomes during antiviral therapy for chronic hepatitis C. Journal of psychosomatic research, 71(5), pp. 349-356.

Fivecoat, H. C. et al. (2018). Social support predicts self-care confidence in patients with heart failure. European Journal of Cardiovascular Nursing 17(7), pp. 598-604. https://doi.org/10.1177/1474515118762800.

Giangrasso, B. y Casale, S. (2014). Psychometric properties of the Medical Outcome Study Social Support Survey with a General Populations Sample of Undergraduate Students. Social Indicators Research, 116(1), pp. 185197.

Gottlieb, H. H. (1983). Social support strategies: Guidelines for mental health practice. Sage Publications.

Grass, J. J. (2013). Emotion regulation: taking stock and moving forward. Emotion, 13(3), pp. 359-365.

Hyrkäs, K. et al. (2003). Validating an instrument for clinical supervision using an expert panel. International Journal of nursing studies, 40(6), pp. $619-625$.

House, J. S. (1981). Work stress and social support. Addison-Wesley.

Juárez, F. (2008). Lejos del corazón. Para los que se van a otro lugar y los que se quedan. Grijalbo.

Khaledi, G. H. et al. (2014). Social support and self-care behavior among heart failure patients: is there any relationship. Journal of Biology and Today's World, 3(9), pp. 194-197.

Khuong, L. Q. et al. (2018). Psychometric properties of the medical outcomes study: social support survey among methadone maintenance patients in Ho Chi Minh City, Vietnam: a validation study. Substance Abuse Treatment, Prevention and Policy, 13(1), pp. 1-8. 
Leung, J. et al. (2014). Social support and health-related quality of life in women with breast cancer: a longitudinal study. Psycho-oncology, 23(9). https://doi.org/10.1002/pon.3523.

Londoño, N. H. et al. (2012). Validación en Colombia del cuestionario MOS de apoyo social. International Journal of Psychological, 5(1), pp. 142-150. http://www.redalyc.org/pdf/2990/299023539016.pdf.

Mahnaz, J. F. et al. (2015). Comparing social support and social anxiety between mother of children with special needs and mothers of normal children. Knowledge y research in applied psychology, 16(2), pp. 43-52.

Martínez, A. E. et al. (2014). Adaptación y validación del Cuestionario MOS de Apoyo Social en pacientes mexicanos con VIH+. Revista Latinoamericana de Medicina Conductual, 4(2), pp. 93-101.

Mikulic, I. (2015). Construcción y adaptación de pruebas psicológicas. Universidad de Buenos Aires, Argentina. https://comenio.files.wordpress.com/2007/08/construccion-y-adaptacion.pdf.

Moser, A. et al. (2012). The eight-item modified Medical Outcomes Study Social Support Survey: psychometric evaluation showed excellent performance. Journal of Clinical Epidemiology, 65(10), pp. 1107-1116.

Naranjo, L. F. y Guerrero, M. (2013). Intraductucbilidad de las metáforas del español a la lengua de señas colombiana. Mutatis Mutandis, 6(2), pp. 477-486.

Quiceno, Y. y Arbeláez, K. (2016). Desafíos de las aulas para sordos y calidad educativa (tesis de licenciatura). Universidad de Antioquia, Medellín.

Ramada, J. M. et al. (2013). Adaptación cultural y validación de cuestionarios de salud: revisión y recomendaciones metodológicas. Salud Pública de México, 55(1), pp. 57-66. http://www.scielo.org.mx/scielo.php?script=sci_ar ttext\&pid=S0036-36342013000100009.

Recine, N. et al. (2018). Maternal adverse childhood experiences and antepartum risks: the moderating role of social support. Archives of women's mental health, 21(6), pp. 663-670.

Robitaille, A. et al. (2018). Psychometric properties, factorial structure, and measurement invariance of the English and French versions of the Medical Outcomes Study social support scale. Stadistics Canada, 22(2), pp. 33-40.

Rutter, M. (2007). Resilience, competency, and coping. Child abuse y Neglect, 31, pp. 205-209.

Sherbourne, C. D. y Stewart, A. (1991). The MOS social support survey. Social Science y Medicine, 32(6), pp. 705-714.

Shyu, Y. et al. (2006). Psychometric testing of the social support survey on a Taiawanese Sample. Nursing Research, 55(6), pp. 411-417.

Sindeaux, M.B. et al. (2016). Parental stress and social support of caregivers of children with cerebral palsy. Paideia (Ribeirao Preto), 26(64), pp. 207-214. https://dx.doi.org/10.1590/1982-43272664201608.

Soares, A. et al. (2012). Validation of the Brazilian Portuguese versión of the Medical Outcomes Study-Social Support Survey in Hodgkin's Lympoma survivors. Supportive Care in Cancer, 20(8), pp 1895-1900.

Thompson, T. et al. (2013). Perceived social support chance in patients with early stage breast cancer and controls. Health Psychology, 32(8), pp. 886-895.

Vargas, A. N. y Coria, K. (2017). Estrés postraumático. Tratamiento basado en la terapia de aceptación y compromiso. Manual Moderno.

Yu, D. S. F. et al. (2004). Psychometric testing of the Chinese version of the medical outcomes study social support survey (MOS-SSS-C). Research in nursing y health, 27(2), pp. 135-43. 
Nora Helena Londoño Arredondo • Luisa Fernanda Suarez Monsalve • Yamile Restrepo Restrepo •

Yeisiri Amaya Montoya • Yonathan Alexander Martínez • Damaris Torres Castro • Yolmer Daniel Peña Cordero •

Maite Catalina Agudelo Cifuentes

Yu, Y. et al. (2015). Psychometric testing of the Chinese version of the Medical Outcomes Study Support Survey among people living with HIV/AIDS in China. Applied Nursing Research, 28(4), pp. 328-333. 


\section{Anexo 1}

Cuestionario MOS (Sherbourne y Stewart, 1991, adaptado para Colombia por Londoño et al., 2012).

\section{Instrucciones}

A continuación, encontrarás algunas preguntas sobre el apoyo o ayuda del que dispones de otras personas.

1. Aproximadamente, ¿cuántos amigos y familiares cercanos tengo? (personas con las que me siento a gusto y con quienes puedo hablar sobre lo que pienso).

Escribo en la casilla el número de amigos y familiares cercanos:

2. Algunas veces, las personas buscan a otras por compañía, ayuda u otro tipo de apoyo. ¿Con qué frecuencia están disponibles para mí cada uno de los siguientes tipos de apoyo si los necesito?

Opciones de respuesta:

\begin{tabular}{|c|c|c|c|c|}
\hline 1. Nunca & 2. Pocas veces & 3. Algunas veces & 4. Muchas veces & 5. Siempre \\
\hline
\end{tabular}

\begin{tabular}{|c|c|c|c|c|c|c|}
\hline 1 & Alguien que me ayude cuando tenga que guardar reposo en cama. & 1 & 2 & 3 & 4 & 5 \\
\hline 2 & Alguien con quien pueda contar para que me escuche cuando necesite hablar. & 1 & 2 & 3 & 4 & 5 \\
\hline 3 & Alguien que me aconseje cuando esté en crisis. & 1 & 2 & 3 & 4 & 5 \\
\hline 4 & Alguien que me lleve al doctor si necesitara ir. & 1 & 2 & 3 & 4 & 5 \\
\hline 5 & Alguien que me demuestre amor y afecto. & 1 & 2 & 3 & 4 & 5 \\
\hline 6 & Alguien con quien paso buenos ratos. & 1 & 2 & 3 & 4 & 5 \\
\hline 7 & Alguien que me dé información para ayudarme a entender una situación. & 1 & 2 & 3 & 4 & 5 \\
\hline 8 & Alguien en quien puedo confiar o hablar sobre mí mismo y mis preocupaciones. & 1 & 2 & 3 & 4 & 5 \\
\hline 9 & Alguien que me abrace. & 1 & 2 & 3 & 4 & 5 \\
\hline 10 & Alguien con quien pueda relajarme. & 1 & 2 & 3 & 4 & 5 \\
\hline 11 & Alguien que me prepare las comidas si no pudiera hacerlo yo misma/o. & 1 & 2 & 3 & 4 & 5 \\
\hline 12 & Alguien de quien realmente desearía recibir un consejo. & 1 & 2 & 3 & 4 & 5 \\
\hline 13 & Alguien con quien pueda hacer algo que me ayude a despejar mi mente. & 1 & 2 & 3 & 4 & 5 \\
\hline 14 & Alguien que me ayude con mis quehaceres domésticos si estuviera enferma/o. & 1 & 2 & 3 & 4 & 5 \\
\hline 15 & Alguien con quien compartir mis mayores preocupaciones y miedos. & 1 & 2 & 3 & 4 & 5 \\
\hline 16 & Alguien a quien acudir para que me sugiera cómo manejar un problema personal. & 1 & 2 & 3 & 4 & 5 \\
\hline 17 & Alguien con quien divertirme. & 1 & 2 & 3 & 4 & 5 \\
\hline 18 & Alguien que entienda mis problemas. & 1 & 2 & 3 & 4 & 5 \\
\hline 19 & Alguien a quien amar y que me haga sentir amado. & 1 & 2 & 3 & 4 & 5 \\
\hline
\end{tabular}




\section{Anexo 2}

Cuestionario de apoyo social a la comunicación y al rol social para comunidad sorda (ComRols) (Londoño y Suárez, 2021).

Instrucciones

A continuación, encontrarás algunas preguntas sobre el apoyo o ayuda del que dispones de otras personas PARTICIPANTE $\mathrm{N}^{\circ}$

Opciones de respuesta:

$$
\text { 1. Nunca }
$$
2. Algunas veces

\section{Siempre}

\begin{tabular}{|c|l|c|c|c|}
\hline \multicolumn{2}{|l|}{ Pregunta en español } & \multicolumn{2}{l|}{ Puntuación } \\
\hline 1 & Alguien que se comunique conmigo en lengua de señas & 1 & 2 & 3 \\
\hline 2 & Alguien que me alerte del peligro & 1 & 2 & 3 \\
\hline 3 & Alguien que me pregunte que quiero hacer en el día & 1 & 2 & 3 \\
\hline 4 & Alguien que me motive para que utilice lengua de señas & 1 & 2 & 3 \\
\hline 5 & Alguien que sea mi intérprete porque sabe lengua de señas & 1 & 2 & 3 \\
\hline 6 & Alguien que me explique cuando no entiendo lo que ocurre o dicen & 1 & 2 & 3 \\
\hline 7 & $\begin{array}{l}\text { Alguien que me acompañe si tengo que hacer vueltas/ diligencias y facilite } \\
\text { la comunicación }\end{array}$ & 1 & 2 & 3 \\
\hline 8 & Alguien que se interese por que estudie/trabaje & 1 & 2 & 3 \\
\hline 9 & Alguien que me comunique dónde estudiar/trabajar & 1 & 2 & 3 \\
\hline 10 & Alguien que me brinde oportunidades académicas/laborales & 1 & 2 & 3 \\
\hline 11 & Alguien que me ayude a buscar convocatorias académicas/laborales & 1 & 2 & 3 \\
\hline 12 & Alguien que conoce mis intereses académicos/laborales & 1 & 2 & 3 \\
\hline
\end{tabular}




\section{Anexo 3. Videos de los cuestionarios}

Cuestionario de Apoyo Social (MOS; Sherbourne y Stewart, 1991).

1. Aproximadamente, ¿cuántos amigos y familiares cercanos tengo? (personas con las que me siento a gusto y con quienes puedo hablar sobre lo que pienso).

Video en YouTube: https://studio.youtube.com/video/sHNPBN_ma38/edi

\begin{tabular}{|c|c|c|}
\hline Pregunta en español & Enlace de youtube & $\begin{array}{l}\text { Nombre del video para } \\
\text { la busqueda en youtube }\end{array}$ \\
\hline $\begin{array}{l}\text { 2. Alguien que me ayude } \\
\text { cuando tenga que guardar } \\
\text { reposo en cama. }\end{array}$ & $\begin{array}{l}\text { https://www.youtube.com/ } \\
\text { watch?v=t4NgM1usy6Q }\end{array}$ & $\begin{array}{l}\text { Cuestionario MOS LSC } \\
\text { avanzado- Pregunta } 2\end{array}$ \\
\hline $\begin{array}{l}\text { 3. Alguien con quien pueda } \\
\text { contar para que me escuche } \\
\text { cuando necesite hablar. }\end{array}$ & $\begin{array}{l}\text { https://www.youtube.com/watch?v=_q37vH- } \\
\text { S6Vkyfeature=youtu.be }\end{array}$ & $\begin{array}{l}\text { Cuestionario MOS LSC } \\
\text { avanzado - Pregunta } 3\end{array}$ \\
\hline $\begin{array}{l}\text { 4. Alguien que me aconseje } \\
\text { cuando esté en crisis. }\end{array}$ & $\begin{array}{l}\text { https://www.youtube.com/watch?v=R30YBo } \\
\text { 4s9lwyfeature=youtu. be }\end{array}$ & $\begin{array}{l}\text { Cuestionario MOS LSC } \\
\text { avanzado - Pregunta } 4\end{array}$ \\
\hline $\begin{array}{l}\text { 5. Alguien que me lleve al } \\
\text { doctor si necesitara ir. }\end{array}$ & $\begin{array}{l}\text { https://www.youtube.com/watch?v=bJ7jJ_ } \\
\text { J3f8Uyfeature=youtu.be }\end{array}$ & $\begin{array}{l}\text { Cuestionario MOS LSC } \\
\text { avanzado - Pregunta } 5\end{array}$ \\
\hline $\begin{array}{l}\text { 6. Alguien que me demuestre } \\
\text { amor y afecto. }\end{array}$ & $\begin{array}{l}\text { https://www.youtube.com/watch?v=2_ } \\
\text { BbJwzL1jAyfeature=youtu.be }\end{array}$ & $\begin{array}{l}\text { Cuestionario MOS LSC } \\
\text { avanzado - Pregunta } 6\end{array}$ \\
\hline $\begin{array}{l}\text { 7. Alguien con quien paso } \\
\text { buenos ratos. }\end{array}$ & $\begin{array}{l}\text { https://www.youtube.com/watch?v=MmZ_ } \\
\text { YfXjr1Yyfeature=youtu.be }\end{array}$ & $\begin{array}{l}\text { Cuestionario MOS LSC } \\
\text { avanzado - Pregunta } 7\end{array}$ \\
\hline $\begin{array}{l}\text { 8. Alguien que me dé } \\
\text { información para ayudarme a } \\
\text { entender una situación. }\end{array}$ & $\begin{array}{l}\text { https://www.youtube.com/watch?v=p6QjhTw } \\
\text { v22gyfeature=youtu.be }\end{array}$ & $\begin{array}{l}\text { Cuestionario MOS LSC } \\
\text { avanzado - Pregunta } 8\end{array}$ \\
\hline $\begin{array}{l}\text { 9. Alguien en quien puedo } \\
\text { confiar o hablar sobre mí } \\
\text { mismo y mis preocupaciones. }\end{array}$ & $\begin{array}{l}\text { https://www.youtube.com/ } \\
\text { watch?v=TtyXZgULdR0 }\end{array}$ & $\begin{array}{l}\text { Cuestionario MOS LSC } \\
\text { avanzado - Pregunta } 9\end{array}$ \\
\hline 10. Alguien que me abrace. & $\begin{array}{l}\text { https://www.youtube.com/watch?v=1hWMr } \\
\text { WDm3UUyfeature=youtu.be }\end{array}$ & $\begin{array}{l}\text { Cuestionario MOS LSC } \\
\text { avanzado - Pregunta } 10\end{array}$ \\
\hline $\begin{array}{l}\text { 11. Alguien con quien pueda } \\
\text { relajarme. }\end{array}$ & $\begin{array}{l}\text { https://www.youtube.com/watch?v=BWhfeo } \\
\text { 6F5Fsyfeature=youtu.be }\end{array}$ & $\begin{array}{l}\text { Cuestionario MOS LSC } \\
\text { avanzado - Pregunta } 11\end{array}$ \\
\hline
\end{tabular}




\begin{tabular}{|c|c|c|}
\hline Pregunta en español & Enlace de youtube & $\begin{array}{l}\text { Nombre del video para } \\
\text { la busqueda en youtube }\end{array}$ \\
\hline $\begin{array}{l}\text { 12. Alguien que me prepare } \\
\text { las comidas si no pudiera } \\
\text { hacerlo yo misma/o. }\end{array}$ & $\begin{array}{l}\text { https://www.youtube.com/ } \\
\text { watch?v=IzYxbQnBBy0 }\end{array}$ & $\begin{array}{l}\text { Cuestionario MOS LSC } \\
\text { avanzado - Pregunta } 12\end{array}$ \\
\hline $\begin{array}{l}\text { 13. Alguien de quien } \\
\text { realmente desearía recibir un } \\
\text { consejo. }\end{array}$ & $\begin{array}{l}\text { https://www.youtube.com/watch?v=86R6- } \\
\text { PunqEA }\end{array}$ & $\begin{array}{l}\text { Cuestionario MOS LSC } \\
\text { avanzado - Pregunta } 13\end{array}$ \\
\hline $\begin{array}{l}\text { 14. Alguien con quien pueda } \\
\text { hacer algo que me ayude a } \\
\text { despejar mi mente. }\end{array}$ & $\begin{array}{l}\text { https://www.youtube.com/watch?v=86R6- } \\
\text { PunqEAyfeature=youtu.be }\end{array}$ & $\begin{array}{l}\text { Cuestionario MOS LSC } \\
\text { avanzado - Pregunta } 14\end{array}$ \\
\hline $\begin{array}{l}\text { 15. Alguien que me ayude } \\
\text { con mis quehaceres } \\
\text { domésticos si estuviera } \\
\text { enferma/o. }\end{array}$ & $\begin{array}{l}\text { https://www.youtube.com/watch?v=jLpiiqFX } \\
\text { zwlyfeature=youtu.be }\end{array}$ & $\begin{array}{l}\text { Cuestionario MOS LSC } \\
\text { avanzado - Pregunta } 15\end{array}$ \\
\hline $\begin{array}{l}\text { 16. Alguien con quien } \\
\text { compartir mis mayores } \\
\text { preocupaciones y miedos. }\end{array}$ & $\begin{array}{l}\text { https://www.youtube.com/ } \\
\text { watch?v=jHR4_OLq0Eo }\end{array}$ & $\begin{array}{l}\text { Cuestionario MOS LSC } \\
\text { avanzado - Pregunta } 16\end{array}$ \\
\hline $\begin{array}{l}\text { 17. Alguien a quien acudir para } \\
\text { que me sugiera cómo manejar } \\
\text { un problema personal. }\end{array}$ & $\begin{array}{l}\text { https://www.youtube.com/ } \\
\text { watch?v=jHR4_0Lq0Eo }\end{array}$ & $\begin{array}{l}\text { Cuestionario MOS LSC } \\
\text { avanzado - Pregunta } 17\end{array}$ \\
\hline $\begin{array}{l}\text { 18. Alguien con quien } \\
\text { divertirme. }\end{array}$ & $\begin{array}{l}\text { https://www.youtube.com/watch?v=kVUtEZg } \\
\text { jc0Ayfeature=youtu.be }\end{array}$ & $\begin{array}{l}\text { Cuestionario MOS LSC } \\
\text { avanzado - Pregunta } 18\end{array}$ \\
\hline $\begin{array}{l}\text { 19. Alguien que entienda mis } \\
\text { problemas. }\end{array}$ & $\begin{array}{l}\text { https://www.youtube.com/ } \\
\text { watch?v=GYSHDu5Z8il }\end{array}$ & $\begin{array}{l}\text { Cuestionario MOS LSC } \\
\text { avanzado - Pregunta } 19\end{array}$ \\
\hline $\begin{array}{l}\text { 20. Alguien a quien amar y } \\
\text { que me haga sentir amado. }\end{array}$ & $\begin{array}{l}\text { https://www.youtube.com/watch?v=5fy } 1 \mathrm{vdu} \\
\text { OaLQyfeature=youtu.be }\end{array}$ & $\begin{array}{l}\text { Cuestionario MOS LSC } \\
\text { avanzado - Pregunta } 20\end{array}$ \\
\hline
\end{tabular}




\begin{tabular}{|c|c|c|}
\hline \multicolumn{3}{|l|}{ Comunicación y ejecución del rol social } \\
\hline Pregunta en español & Enlace en Youtube & $\begin{array}{l}\text { Nombre del video para la } \\
\text { búsqueda en Youtube }\end{array}$ \\
\hline $\begin{array}{l}\text { 1. Alguien que se comunique } \\
\text { conmigo en lengua de señas }\end{array}$ & $\begin{array}{l}\text { https://www.youtube.com/ } \\
\text { watch?v=iFbv42QBm5I }\end{array}$ & $\begin{array}{l}\text { Cuestionario COMROLS } \\
\text { LSC avanzado - Pregunta } 1\end{array}$ \\
\hline $\begin{array}{l}\text { 2. Alguien que me alerte del } \\
\text { peligro }\end{array}$ & $\begin{array}{l}\text { https://www.youtube.com/ } \\
\text { watch?v=iFbv42QBm5I }\end{array}$ & $\begin{array}{l}\text { Cuestionario COMROLS } \\
\text { LSC avanzado - Pregunta } 2\end{array}$ \\
\hline $\begin{array}{l}\text { 3. Alguien que me pregunte que } \\
\text { quiero hacer en el día }\end{array}$ & $\begin{array}{l}\text { https://www.youtube.com/watch?v=iFbv } \\
\text { 42QBm5lyfeature=youtu.be }\end{array}$ & $\begin{array}{l}\text { Cuestionario COMROLS } \\
\text { LSC avanzado - Pregunta } 3\end{array}$ \\
\hline $\begin{array}{l}\text { 4. Alguien que me motive para } \\
\text { que utilice lengua de señas }\end{array}$ & $\begin{array}{l}\text { https://www.youtube.com/ } \\
\text { watch?v=jWLSHsHDlgs }\end{array}$ & $\begin{array}{l}\text { Cuestionario COMROLS } \\
\text { LSC avanzado - Pregunta } 4\end{array}$ \\
\hline $\begin{array}{l}\text { 5. Alguien que sea mi intérprete } \\
\text { porque sabe lengua de señas. }\end{array}$ & https://youtu.be/bqBIVVdONOo & \begin{tabular}{|l} 
Cuestionario COMROLS \\
LSC avanzado - Pregunta 5
\end{tabular} \\
\hline $\begin{array}{l}\text { 6. Alguien que me explique } \\
\text { cuando no entiendo lo que } \\
\text { ocurre o dicen }\end{array}$ & $\begin{array}{l}\text { https://www.youtube.com/watch?v=bqBI } \\
\text { WVdONOoyfeature=youtu.be }\end{array}$ & $\begin{array}{l}\text { Cuestionario COMROLS } \\
\text { LSC avanzado - Pregunta } 6\end{array}$ \\
\hline $\begin{array}{l}\text { 7. Alguien que me acompañe } \\
\text { si tengo que hacer vueltas/ } \\
\text { diligencias y facilite la } \\
\text { comunicación. }\end{array}$ & $\begin{array}{l}\text { https://www.youtube.com/ } \\
\text { watch?v=rGcQSYxt_Dgyfeature=youtu. } \\
\text { be }\end{array}$ & $\begin{array}{l}\text { Cuestionario COMROLS } \\
\text { LSC avanzado - Pregunta } 7\end{array}$ \\
\hline $\begin{array}{l}\text { 8. Alguien que se interese por } \\
\text { que estudie/trabaje }\end{array}$ & $\begin{array}{l}\text { https://www.youtube.com/watch?v=0i9p } \\
\text { QzHlyl4yfeature=youtu.be }\end{array}$ & $\begin{array}{l}\text { Cuestionario COMROLS } \\
\text { LSC avanzado - Pregunta } 8 \\
\end{array}$ \\
\hline $\begin{array}{l}\text { 9. Alguien que me comunique } \\
\text { dónde estudiar/trabajar }\end{array}$ & $\begin{array}{l}\text { https://www.youtube.com/ } \\
\text { watch?v=WleWE-p2llcyfeature=youtu.be }\end{array}$ & $\begin{array}{l}\text { Cuestionario COMROLS } \\
\text { LSC avanzado - Pregunta } 9 \\
\end{array}$ \\
\hline $\begin{array}{l}\text { 10. Alguien que me brinde } \\
\text { oportunidades académicas/ } \\
\text { laborales }\end{array}$ & $\begin{array}{l}\text { https://www.youtube.com/watch?v=mVoj } \\
\text { Z2KO5Xkyfeature=youtu.be }\end{array}$ & $\begin{array}{l}\text { Cuestionario } \text { COMROLS } \\
\text { LSC avanzado - Pregunta } \\
10\end{array}$ \\
\hline $\begin{array}{l}\text { 11. Alguien que me ayude } \\
\text { a buscar convocatorias } \\
\text { académicas/laborales }\end{array}$ & $\begin{array}{l}\text { https://www.youtube.com/watch?v=6Cg } \\
\text { Owa1EAi4yfeature=youtu.be }\end{array}$ & $\begin{array}{l}\text { Cuestionario COMROLS } \\
\text { LSC avanzado - Pregunta } \\
11\end{array}$ \\
\hline $\begin{array}{l}\text { 12. Alguien que conoce mis in- } \\
\text { tereses académicos/laborales }\end{array}$ & $\begin{array}{l}\text { https://www.youtube.com/watch?v=bqBI } \\
\text { WVdONOoyfeature=youtu.be }\end{array}$ & $\begin{array}{l}\text { Cuestionario COMROLS } \\
\text { LSC avanzado - Pregunta } \\
12\end{array}$ \\
\hline
\end{tabular}

